\title{
Capacitive Sensing
}

\author{
Georg Brasseur \\ Graz University of Technology, Dep. of Electrical Measurement \& Measurement Signal Processing \\ TU-Graz, Inst. E438, Kopernikusgasse 24, A-8010 Graz, Austria
}

\section{Introduction}

Capacitive sensors determine the electric field distribution in a measurement volume to capture the measurand like a geometrical quantity, a mass distribution or a volume stream. Since a long time scientists have worked in this field to design highly reliable capacitive sensors [1] but could not obtain a visible market penetration. Thanks to microelectronics, capacitive sensing has become more and more popular in the recent years even for failure critical systems in an industrial and automotive environment. State-ofthe-art airbag systems and electronic stability programs for vehicles would almost be impossible without capacitive sensing. Today's capacitive sensing applications include touch pads, proximity and rotary switches, occupant detection, parking aids, inclination, encapsulated and not encapsulated position, nano-positioning, tank fill level, film and layer thickness, oil quality, smart textiles, flow measurement, electrical capacitance Tomography.

Some achievements from mobile communications contribute to capacitive sensing like low noise amplifiers, low power analogue-to-digital converters, frequency hopping spread spectrum and time division multiplex algorithms. Companies would have never spent money to design such optimised structures for the use in capacitive sensors as the sales volumes are much too small compared to mobile communications. As such building blocks are available now CDCs get a remarkable thrust from mobile communications and the semiconductor platform grows fast for small, reliable, robust and low cost capacitive sensing circuits tailored to an industrial environment.

A huge benefit of capacitive sensing is the simplicity of the front-end: the sensing elements are numerous conductive areas attached to an insulator. The sensor electronics measures the capacitances between these areas and the electronics calculates each measurement result from numerous capacitance measurements. As the conductive areas can be made out of almost all types of materials the sensing elements can be fabricated in a way to be both, inexpensive and robust.

One challenge of capacitive sensing are the small capacitance values to be measured between corresponding plates, usually in the $\mathrm{pF}$ to $\mathrm{fF}$ range with a resolution as low as $\mathrm{aF}$. Consequently, the measurement electronics has to be located as close as possible to the transmitter and receiver electrodes to avoid leakage effects and disturbances from the environment. Typically, the front-end electronics, commonly called "Capacitance-to-Digital Converters (CDC)", encompasses the entire analogue and digital signal processing circuitry as well as transmitters and output drivers to communicate with a master [2].

The aim of the paper is to give a summary of already published challenges and answers related to electrode topologies, sensor circuitries and algorithms for robust capacitive sensors [3] tailored to an industrial environment.

\section{Basic Considerations}

Usually, all inputs of the sensor front-end circuitry need a huge dynamic range with excellent linearity. Furthermore, the inputs have to cope with small signal levels and with a poor signal-to-noise ratio as the geometric and dielectric properties of the measurement capacitances may vary largely in and with the application.

All circuit pins connected to sensing electrodes are subject of Electro Static Discharge (ESD) and disturbing electric fields up to $200 \mathrm{~V} / \mathrm{m}$ in a frequency range from $100 \mathrm{kHz}$ up to $2 \mathrm{GHz}$ and must therefore be reliably protected against overload currents (Electro Magnetic Compatibility, EMC).

Conductive contamination, moisture and dew may come in contact with the sensor. A hermetically encapsulated housing solves this "contamination problem" but not with sensors where the environment needs direct access to the sensing elements, like with angular sensors [4] and torque sensors [5]. Consequently, the transducer can not be hermetically encapsulated and some other precautions have to be provided to cope with the "contamination problem" as well as with the ESD and EMC problem. 
A simple solution to cope with both, the "contamination" and the ESD/EMC problem, can be adopted from radio transmission by implementing a carrier frequency system with passive components at the input forming a resonant circuit (see Fig. 1). The passive components $R, L, C$ of the resonant circuit have an intrinsically high dynamic range, are able to cope with overloads and the resonant circuit amplifies the input voltage noiseless by a gain equivalent to the quality factor.

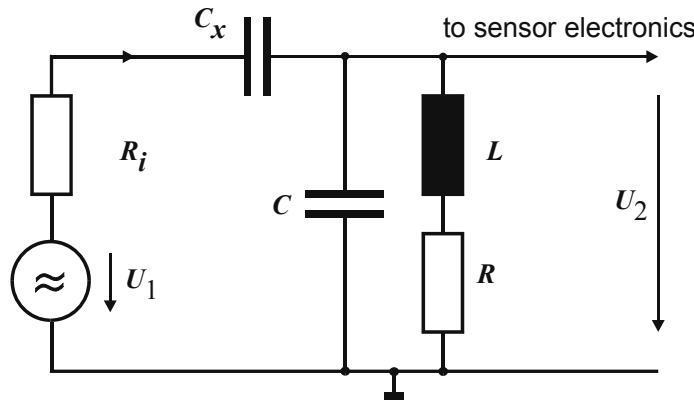

a)

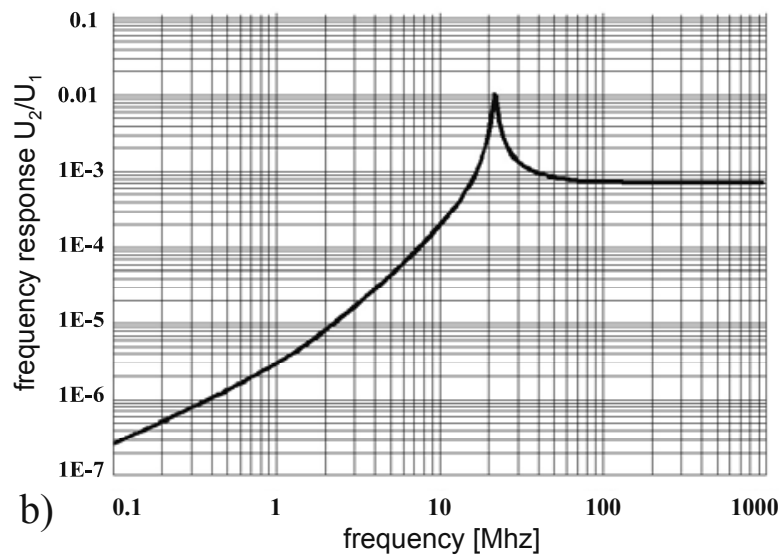

b) Frequency response of the transfer

Fig. 1 a) Equivalent circuit of the front-end of a carrier frequency
characteristics $U_{2} / U_{1}$ of the front-end resonant circuit [13].

$U_{1} \ldots$ Excitation signal; $U_{2} \ldots$ Signal to the $1^{\text {st }}$ amplifier; $C_{\mathrm{x}} \ldots$ Coupling capacitance; $R_{\mathrm{i}} \ldots$ Transmitter impedance; R, L, C... Resonator components.

The measurement set-up of a capacitive sensor uses at least one transmitter electrode to excite an electric field with respect to a reference potential and at least one receiver electrode to capture the electric potential within the measurement volume. The input impedance of the amplifier connected to the receiver electrode has to be as high as possible else wise the recorded electrical potential would differ from the true value without the electronics connected. Amplifiers and connecting cables operated at high measurement frequencies do not have a high input impedance. Therefore, measuring displacement currents instead of field potentials (voltages) is more practical and precise but implies a fixed potential, usually reference or ground, at the receiver electrode.

The magnitude of the received displacement current is defined by the geometry and the material properties within the measurement volume, by the location of the electrodes and particularly by the electrical excitation signals. Measurement errors will be introduced by the excitation signal as well as by stray capacitances and imperfections of the receiving electronics including amplifiers (I/U-converter), rectification, filtering and analogue-to-digital (AD) conversion (see Fig. 8). The transmission of flawless excitation signals is possible with a simple electronics but it is technically not feasible to build an almost perfect receiving circuitry for the measurement of capacitances in the $\mathrm{pF}$ to fF range. Therefore, it is advantageous to use only one receiver and multiple transmitters. Gain and offset errors will cancel out if the linearity of the receiving electronics is guaranteed and if a ratiometric measurement algorithm is implemented [3] and [6]. Such algorithms rely on quantities derived from differences and quotients of the measured capacitances instead of using absolute measurement values: Let $S$ be a variable used in the measurement algorithm than $S$ should be calculated from the measured displacement currents as being proportional to the capacitances $C 1$ and $C 2$ with a formula like: $S=(C 1-C 2) /(C 1+C 2)$.

Lessons learned from the basic considerations:

1) Use a high frequency carrier frequency system with lock-in-amplifier technique and a passive resonant circuit at the front-end to get the following benefits:

- good signal-to-noise ratio even in an environment with conductive contaminations like water, moisture, dew and staining,

- noiseless gain of the received signal by the resonator's quality factor and

- powerful filter to fight ESD and EMC problems .

2) Only use one receiver with good linearity across the required dynamic range but multiple transmitters.

3) Capture displacement currents with a receiver electrode kept at reference or ground potential instead of measuring field potentials (voltages) with high input impedance amplifiers.

4) Implement a ratiometric measurement algorithm. 


\section{Coupling Modes}

The rotor material of a capacitive sensor affects the coupling capacitance between two or more electrodes (see Fig. 2). The capacitance $C$ between transmitter and receiver increases if the conductive rotor is insulated (case a) or decreases if the conductive rotor is grounded (case b). On the other hand, a dielectric rotor will increase the transmitter-to-receiver capacitance (case c).

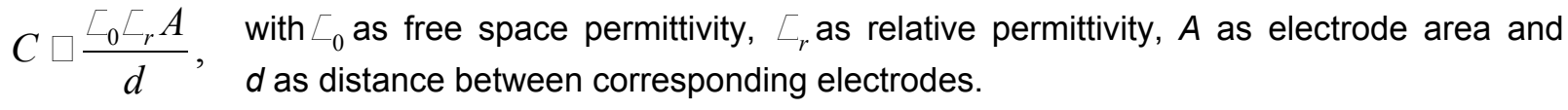

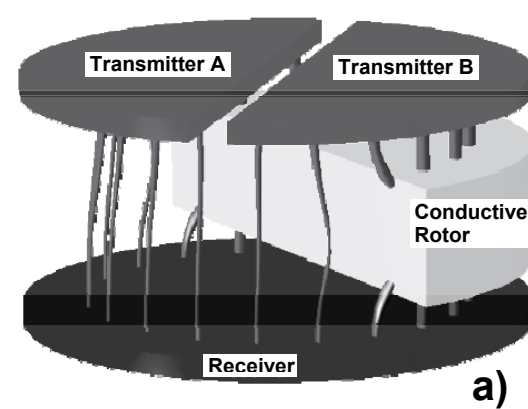

a)

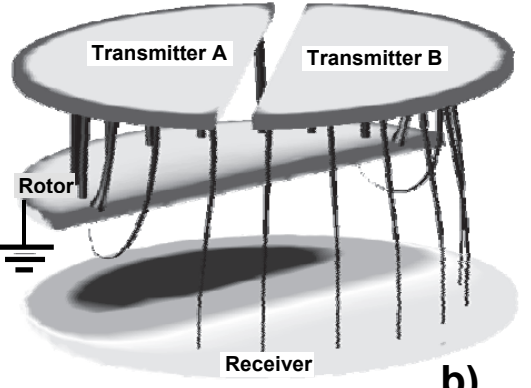

b)

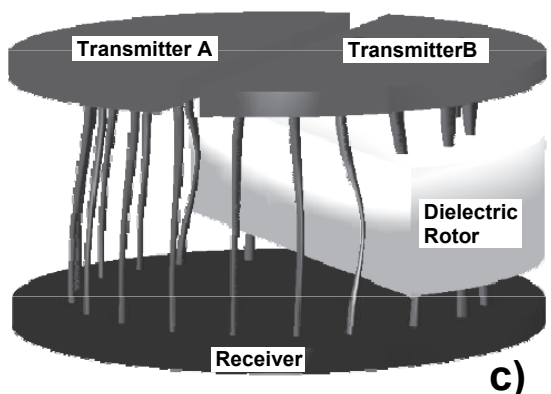

c)

Fig. 2 Coupling modes of capacitive sensors from [7]: a) Conductive coupling, b) Conductive shielding and c) Dielectric coupling

The following example of a more complex electrode structure used in a seat occupant sensing system published in [7] and [8] demonstrates that coupling and shielding mode of operation may merge unexpectedly depending on the seating position of a human.

An automobile seat equipped with the sensing system is displayed in Fig. 3. It uses a single receiver electrode and 11 transmitter electrodes. The proposed system provides information about the presence, type (distinguishes between adult and child occupants), and position of an occupant. As the system uses a single receiver electrode, the calibration of the system is much simpler in comparison to systems with multiple receiver electrodes.

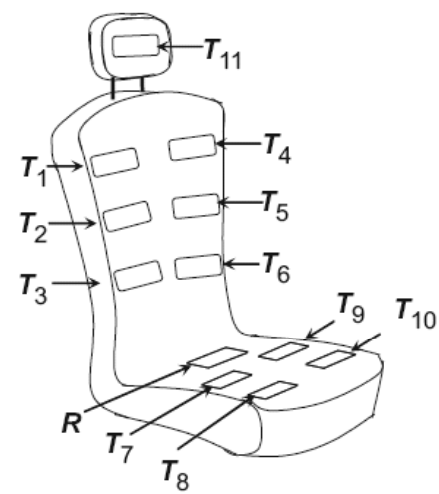

Fig. 3 Automobile seat equipped with the proposed structure of capacitive sensing electrodes from [8]. Electrode $R$ in the sitting area is the common receiver, while electrodes $T 1, T 2, \ldots, T 11$ are transmitters.

An electrical equivalent circuit of the sensor system with a human body in the vicinity of the sensor electrodes is shown in Fig. 4. For simplicity, only one transmitter electrode $T 1$ and the receiver electrode $R$ are used to explain the principle of operation.

Fig. 4 Pictorial representation of the electric field lines due to the presence of a human in the vicinity (shielding and coupling mode).

For clarity, the electric field lines from transmitter to ground are not shown. Similarly, the distributed capacitances between the human body and ground are represented by a lumped capacitance $C_{\mathrm{GH}}$.

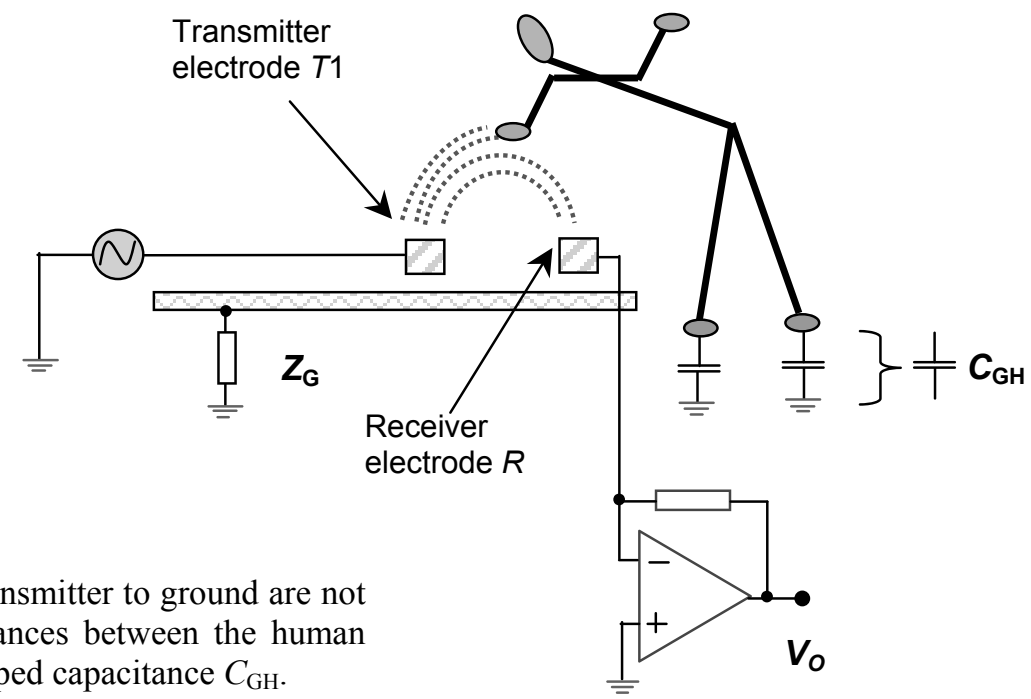


Let us consider that the transmitter $T 1$ is kept at an electric potential and the receiver $R$ is at circuit ground potential. Then, there will be electric field lines emanating from the transmitter $T 1$ to the receiver $R$. Consider, as shown in Fig. 5, that only a small portion of the sensing volume is now occupied by the human body. In such a case, the human body shields some of the electric field lines as shown in Fig. 4. Consequently, the signal received by $R$ and, hence, the output signal $V_{O}$ will be reduced compared to a vacant condition. In this mode, the capacitances $C_{\mathrm{TH}}$ and $C_{\mathrm{RH}}$ will be low in value as compared to $C_{\mathrm{GH}}$.

On the other hand, when the human body comes very close or is between the transmitter and receiver (refer to Fig. 6 ), $C_{\mathrm{TH}}$ and $C_{\mathrm{RH}}$ become much larger than $C_{\mathrm{GH}}$, and hence, $I_{\mathrm{TH}}$ will be much lower compared to $I_{\mathrm{TR}}$. Thus, the current $I_{\mathrm{TR}}$ from the transmitter to the receiver electrode increases in comparison with its value during the shielding mode. This is referred to as the coupling mode of operation, and it is shown using an equivalent circuit in Fig. 6 .

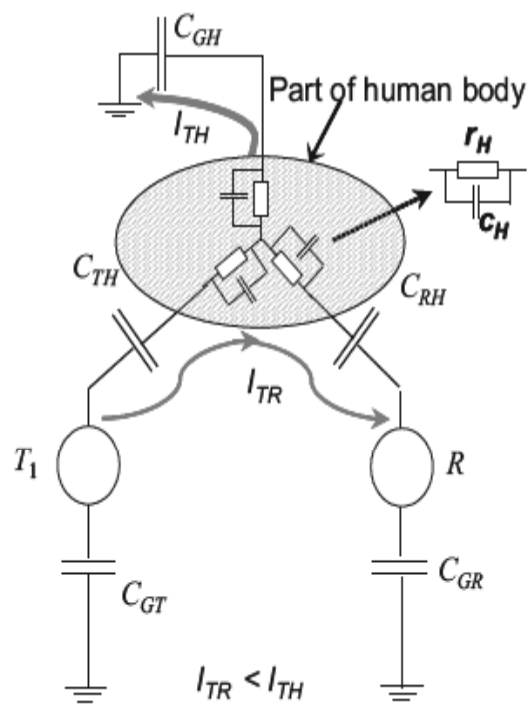

$C_{\mathrm{GH}}$ between human body and ground

$C_{\mathrm{TH}}$ between Transmitter electrode and human body

$C_{\mathrm{RH}}$ between Receiver electrode and human body

$C_{\mathrm{GT}}$ between Transmitter electrode and ground

$C_{\mathrm{GR}}$ between Receiver electrode and ground

$r_{\mathrm{H}}$ resistance of human body part

$C_{\mathrm{H}}$ capacitance of human body part

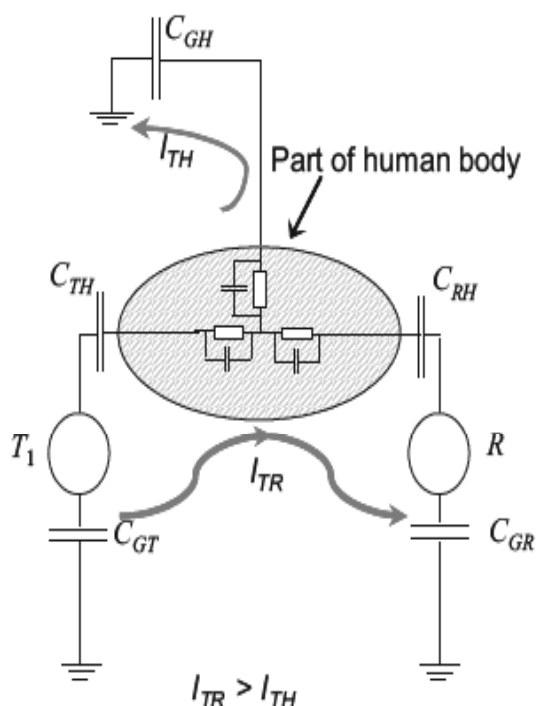

Fig. 5 Equivalent circuit showing the shielding effect by a human body. In shielding mode, current $I_{\mathrm{TH}}$ increases as the human body enters the vicinity of the sensing electrodes. Hence, the signal received by $R$ is reduced. $\quad$ Source: [8]

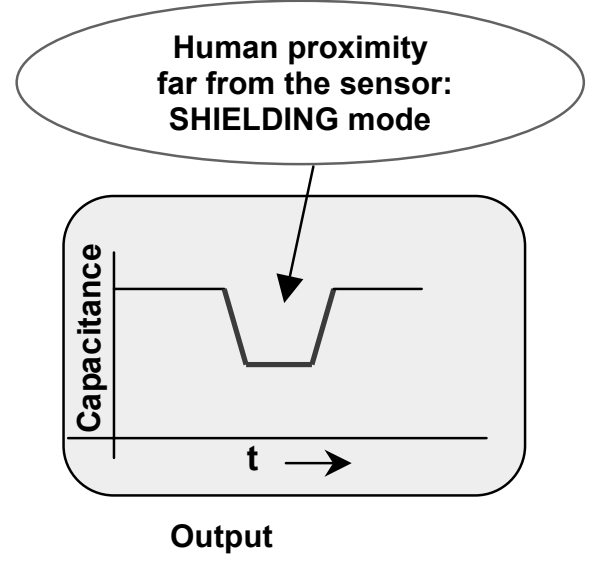

Fig. 5a Human proximity shielding mode:
Fig. 6 Capacitances $C_{\mathrm{TH}}$ and $C_{\mathrm{RH}}$ are larger than $C_{\mathrm{GH}}$ when the human body is very close to the sensing electrodes. Under this condition, the coupling effect is more dominant than the shielding effect.

Source: [8]

Above example of a seat occupant sensing system demonstrates that a two electrode set-up may not be clear without ambiguity. Shielding mode and coupling mode will compensate at a distance, and hence, the proximity of a human would not be recognized. Fig. 7 shows this fact in a planar two electrode set-up. 
The receiver is located beside the transmitter and the measured charge is equivalent for a distance between the electrodes surface and the solid dielectric for around $0.7 \mathrm{~mm}$ and $3.3 \mathrm{~mm}$ respectively.

Additional ambiguity is introduced by the size and material properties of the subject matter to be detected. A huge item a long way off with a good conductivity and/or a high permittivity may give the same reading as a small object with a poor conductivity and/or low permittivity close-by.

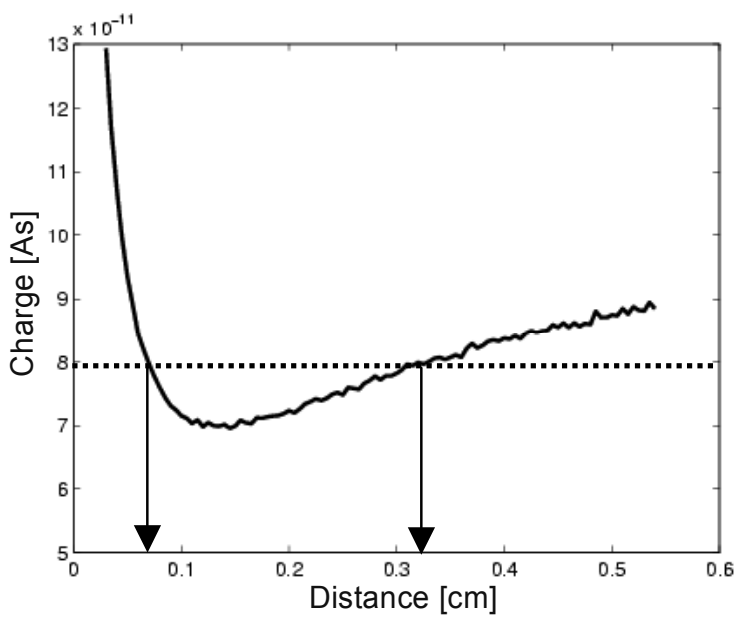

Fig. 7 Received charge in a planar two electrode set-up with respect to the distance between the electrodes surface and the solid dielectric.

Let us summarize that one receiver and at least two transmitters have to be used to cope with the ambiguity problem. Thus, the seat occupant sensing system uses multiple transmitters to obtain the unparalleled classification characteristics published in [7] and [8].

\section{Sensor Electronics}

Beside the "Lessons learned from the basic considerations" outlined in section 2. some more remarks concerning the sensor electronics follow.

The challenge of the carrier frequency system with a lock-in amplifier is the sensitivity to electromagnetic interference in the pass band of the resonant circuit. However, transmission technology has shown reliable solutions to this problem by introducing e.g. frequency hopping or direct sequence spread spectrum techniques [9].

Let us consider instead of a carrier frequency system the use of a charge amplifier or a switched capacitor relaxation oscillator or a $R C$-oscillator. On one hand we would obtain a more simple and low power electronics but at the disadvantage of several constraints: The resonant circuit at the front-end has to be omitted. Hence, coping with ESD and EMC is much more severe. Interference signals like from the mains and from radio transmission can hardly be handled in a not encapsulated housing and without passive filtering at the front-end. Furthermore, the total transmission sequence has to be DC-offset free.

Fig. 8 taken from [10] presents the front-end of a carrier frequency measurement system. The input current is multiplied by two signals that have the same frequency as the excitation signal but are shifted by 90 degrees in phase. The average values of the resulting signals correspond to the real (I) and imaginary (Q) signal parts and, thus, to the capacitance and conductance between the sensor electrodes.

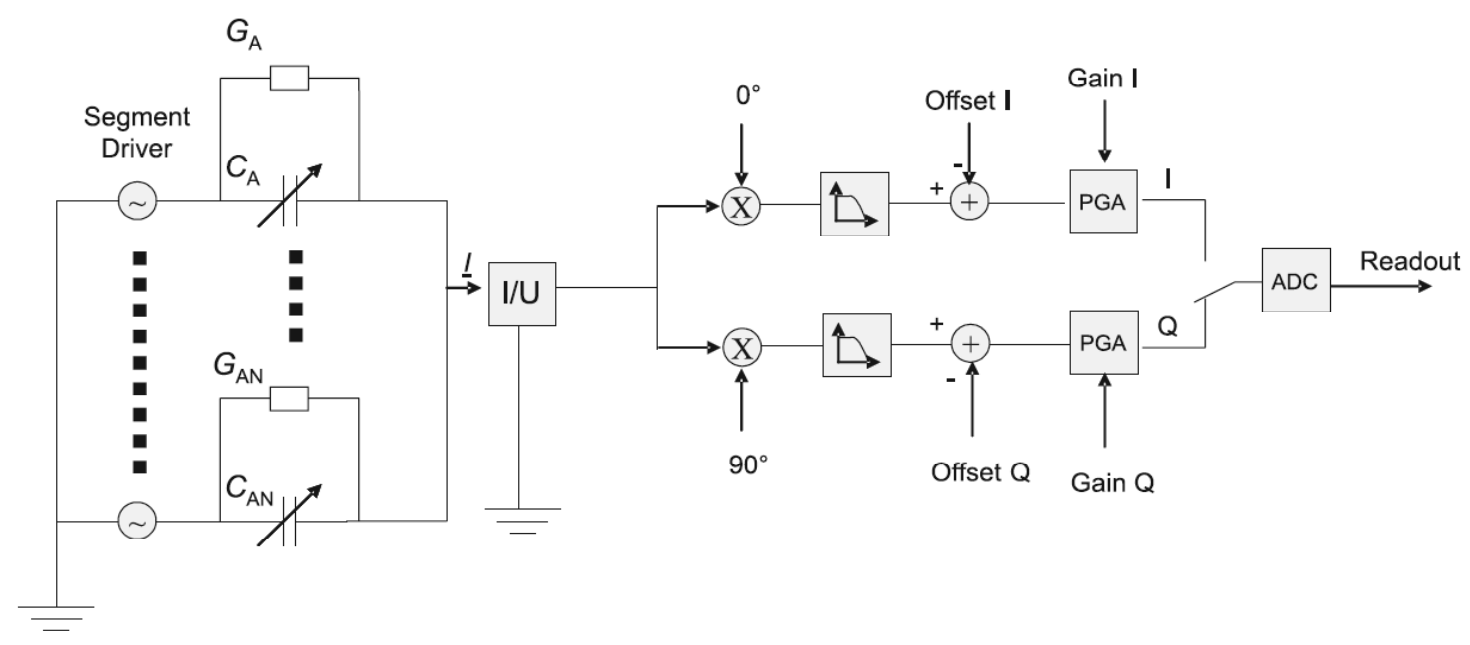

Fig. 8 Principle of a carrier frequency measurement system for an array of transmitter electrodes and a common receiver electrode with $\mathrm{I} / \mathrm{Q}$ demodulation and offset compensation [10]. 


\section{Measurement Algorithms}

Although capacitive sensors may achieve high accuracy under laboratory conditions, they suffer from performance degradation due to environmental influences in the absence of appropriate counter measures. Therefore, measurement algorithms have to be implemented in the sensor to cope with environmental influences. Following is an example of the algorithm impact to the accuracy of a fluidic inclinometer used to trigger an online condition monitoring system of a crane to prevent turnover [11].

In the parameter screening the fill level was found to be most relevant for the accuracy due to its comparatively high variance over temperature and time. Therefore, the authors of [11] considered for the reconstruction of the inclination different estimators. In Fig. 9 the maximum mean square errors for different estimators are compared. With an optimised algorithm the error of the sensor could be reduced by about $50 \%$ despite temperature and aging influences.

Fig. 9 Comparison of different estimators. 'CRLB' denotes the Cramer-Rao Lower Bound, 'Ratio' denotes the classical ratiometric algorithm, 'DFT' denotes an estimator based on the Discrete

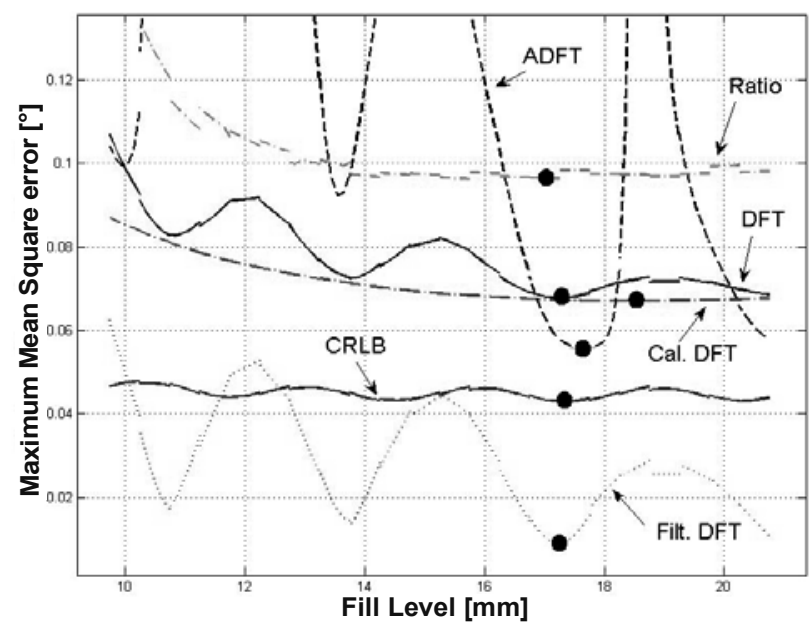

Fourier Transform (DFT), 'Cal. DFT' marks a DFT estimator with calibration, 'Filt. DFT' marks a DFT estimator with infinite averaging and 'ADFT' denotes a DFT estimator with phase averaging of harmonics. The marks indicate the location of the optimum design. The error is reduced by about $50 \%$, mainly due to bias reduction.

\section{Conclusion}

The paper presents a brief overview of today's capacitive sensing technologies. In particular, challenges and answers concerning coupling modes, sensor electronics and measurement algorithms are discussed. Each chapter gives examples taken from literature of successfully implemented capacitive sensor applications and conclusions are drawn from the presented approaches.

\section{References}

[1] E. C. Heerens, "Basic Principles in Designing Highly Reliable Multiterminal Capacitor Sensors and Performance of some Laboratory Test Models". Sensors and Actuators, vol. 3, pp. 137-148, 1982/83.

[2] Analog Device, “AD7147, CapTouch Programmable Controller for Single-Electrode Capacitance Sensors”. Data Sheet, http://www.analog.com/static/imported-files/Data_Sheets/AD7147.pdf, 24.2.2009.

[3] G. Brasseur, „Design Rules for Robust Capacitive Sensors“. In IEEE Transactions on Instrumentation and Measurement, vol. 52, no. 4, pp. 1261-1265, 2003.

[4] G.W. de Jong, G.C.M. Meijer, K. van der Lingen, J.W. Spronck, A.M. Aalsma and Th.D. Berteles, “A smart capacitive absolute angular-position sensor". Sensors and Actuators, vol. A41-42, pp. 212-216, 1994.

[5] St. Cermak, F. Wandling, W. Zdiarsky, P. Fulmek, G. Brasseur, "Capacitive Sensor for Relative Angle Measurement". In IEEE Conference Proceedings on Instrumentation and Measurement Technology (IMTC/2000), Baltimore Hilton and Towers, Maryland, USA, May 1-4, vol.2, pp. 830-833, 2000.

[6] G. Brasseur, "A Robust Capacitive Angular Position Sensor“. In IEEE Proceedings on Instrumentation and Measurement Technology (IMTC/96), Brussels, Belgium, June 4-6, pp. 1081-1086, 1996.

[7] H. Zangl, Th Bretterklieber, D. Hammerschmidt and T. Werth, "Seat Occupancy Detection Using Capacitive Sensing Technology“. In Confernce Proceedings of the SAE World Congress, Detroit, MI, USA, April 14-17, 2008.

[8] B. George, H. Zangl, Th. Bretterklieber and G. Brasseur, "Seat Occupancy Detection based on Capacitive Sensing”. In print for IEEE Transactions on Instrumentation and Measurement, 2009.

[9] H. Zangl, Th. Bretterklieber, G. Holler, "On the Design of a Fully Differential Capacitive Sensor Front-End Interface Circuit”. In Conference Proceedings of Informationstagung Mikroelektronik, Vienna, Austria, Oct. 15-16, pp 132-136, 2008.

[10] Th. Betterklieber1, H. Zangl, M. Motz, T. Werth, D. Hammerschmidt, "Versatile Sensor Front End for Low-Depth Modulation Capacitive Sensors". In IEEE Conference Proceedings on Instrumentation and Measurement Technology (I2MTC/08), Vancouver Island, Canada, May12-15, 2008.

[11] H. Zangl, Th. Bretterklieber, "Rotor Design for Capacitive Sensors". In IEEE Conference Proceedings on Sensors, Vienna, Austria, Oct. 24-27, pp. 520-523, 2004. 